\title{
Ertugliflozin, renoprotection and potential confounding by muscle wasting. Reply to Groothof D, Post A, Gans ROB et al [letter]
}

\author{
David Z. I. Cherney ${ }^{1}$ (D) $\cdot$ Bernard Charbonnel $^{2}$ • Francesco Cosentino ${ }^{3} \cdot$ Samuel Dagogo-Jack $^{4}$ • Darren K. McGuire ${ }^{5,6}$. \\ Richard Pratley ${ }^{7}$. Weichung J. Shih ${ }^{8,9} \cdot$ Robert Frederich $^{10} \cdot$ Mario Maldonado $^{11}$ • Annpey Pong ${ }^{12}$. \\ Christopher P. Cannon ${ }^{13}$. on behalf of the VERTIS CV Investigators
}

Received: 29 October 2021 / Accepted: 2 November 2021 / Published online: 3 March 2022

(C) Pfizer Inc. and Merck Sharp \& Dohme Corp., a subsidiary of Merck \& Co., Inc., Kenilworth, N.J., U.S.A. 2022

Keywords Body weight $\cdot$ Chronic kidney disease $\cdot$ Ertugliflozin $\cdot$ Estimated glomerular filtration rate $\cdot$ Kidney outcomes · Sodium-glucose cotransporter 2 inhibitor

\section{Abbreviations \\ CKD Chronic kidney disease \\ HF Heart failure \\ SGLT2 Sodium-glucose cotransporter 2 \\ VERTIS CV eValuation of ERTugliflozin effIcacy and Safety CardioVascular outcomes}

To the Editor: We wish to thank Groothof et al for their thoughtful letter regarding the relationship between muscle mass and kidney protection, especially changes in eGFR over time with the use of sodium-glucose cotransporter 2 (SGLT2) inhibitors [1]. As the authors indicate, SGLT2 inhibitors, including ertugliflozin, induce body weight loss of approximately $2-3 \mathrm{~kg}$ in most trials in people with type 2 diabetes mellitus with preserved kidney function. In their letter, Groothof et al raise the hypothesis that this body weight reduction is at least partly accounted for by a 'substantial loss of muscle mass', and that this may partly mimic kidney protection by mediating a decline in serum creatinine through decreased creatinine production. In their scenario, kidney

\footnotetext{
David Z. I. Cherney

david.cherney@uhn.ca

University of Toronto, Toronto, ON, Canada

2 University of Nantes, Nantes, France

3 Unit of Cardiology, Karolinska Institute \& Karolinska University Hospital, Stockholm, Sweden

4 University of Tennessee Health Science Center, Memphis, TN, USA

5 University of Texas Southwestern Medical Center, Dallas, TX, USA

6 Parkland Health and Hospital System, Dallas, TX, USA
}

protection is partly a biochemical epiphenomenon related to decreased creatinine production, rather than preservation of kidney function and solute clearance.

An important consideration against the hypothesis advanced by Groothof et al is the pattern of kidney function change compared with body weight change over time following SGLT2 inhibitor use. SGLT2 inhibitors induce an initial acute haemodynamic effect, characterised by a dip in eGFR, which is typically maximal by 4-8 weeks, followed by a return towards baseline by 12-16 weeks [2]. Body weight loss with SGLT2 inhibitors is quite rapid, with maximal effects being seen in the initial 4-8 weeks that then persist over time; these initial changes are, in large part, accounted for by reduced body water volume [3]. Accordingly, the temporal sequence of eGFR and body weight changes are dyssynchronous and are, therefore, unlikely to be physiologically related.

Beyond this chronological dissociation, existing data have not shown a consistent change in muscle mass in response to SGLT2 inhibition in either direction. In fact, in four [4-7] of the six trials [4-9] with dapagliflozin (which is pharmacologically similar to ertugliflozin) quoted by the authors, there was

AdventHealth Translational Research Institute, Orlando, FL, USA

8 Rutgers School of Public Health, New Brunswick, NJ, USA

9 Rutgers Cancer Institute of New Jersey, New Brunswick, NJ, USA

10 Pfizer Inc., Collegeville, PA, USA

11 MSD Limited, London, UK

12 Merck \& Co., Inc., Kenilworth, NJ, USA

13 Cardiovascular Division, Brigham and Women's Hospital, Harvard Medical School, Boston, MA, USA 
no effect on measures of lean mass [10], whilst a recent active comparator (glibenclamide) trial showed an increase in lean:total body mass ratio with dapagliflozin use [11]. In addition to the inconsistent directional effects on measures of lean body mass, we also wish to emphasise that, even if changes in lean body mass did occur, this variable is composed of several factors, including muscle and water content. Accordingly, in the unlikely scenario that lean mass was affected by the amounts suggested in several trials [10], at least $50 \%$ of a lean mass loss of generally $<1.0 \mathrm{~kg}$ is accounted for by water loss [12]. From a quantitative perspective, this degree of muscle mass loss would not contribute to changes in eGFR in a clinically meaningful way over time.

A third set of observations that make it unlikely that body weight changes (as a surrogate for muscle mass) and kidney protection are related come from other trial cohorts [13-15]. We now know from dedicated trials in patients with kidney disease that SGLT2 inhibitors substantially reduce the risk of chronic kidney disease (CKD) progression, even in patients with baseline eGFR levels as low as $25 \mathrm{ml} \mathrm{min}^{-1}[1.73 \mathrm{~m}]^{-2}$, including those without type 2 diabetes [13, 14]. Importantly, in patients without diabetes and those with eGFR $<30 \mathrm{ml} \mathrm{min}^{-1}[1.73 \mathrm{~m}]^{-2}$, the effects of SGLT2 inhibitors on glycaemic control and body weight are clinically negligible or neutral and, yet, profound kidney protection has been observed (not just based on eGFR decline but also on the number of events of end-stage kidney disease) [14, 15]. Importantly, this protection has been reported in heart failure (HF) trials, which also included individuals with CKD stage 4 and those without diabetes [16]. Although the eValuation of ERTugliflozin effTcacy and Safety CardioVascular outcomes (VERTIS CV) trial (ClinicalTrials.gov registration no. NCT01986881) did not enrol individuals with CKD stage 4 or necessarily include those at risk of CKD progression [17], as the authors point out, it is likely that the same principles apply in VERTIS CV [18].

Regarding the authors' point that 'therapy-related muscle wasting' may conceal the need to start dialysis, changes in body weight and related muscle mass, if present at all, would occur very early following the commencement of SGLT2 inhibitor therapy, and there is no indication that body weight acts as a surrogate for muscle mass changes over time during the course of trials lasting 3-4 years [17]. Specifically, body weight loss plateaus early after initiation of SGLT2 inhibitor therapy and does not continue to decrease with chronic treatment [17]. Accordingly, previous body weight loss that is not progressive does not alter eGFR trends and, therefore, would not have an impact on decision making related to dialysis initiation.

Furthermore, the decision to initiate dialysis is not made based on biochemical factors (i.e. eGFR) alone, and would be made in conjunction with other clinical parameters, such as electrolyte levels and signs and/or symptoms of volume overload [19]. Hence, the authors' suggestion that SGLT2 inhibitors may delay the start of dialysis by mitigating hypervolaemia is plausible, as these therapies reduce the risk of hospitalisation for HF across trials, including VERTIS CV [17], and avoidance of hypervolaemia may be an additional benefit in nephrology practice. Other methods to improve the operating characteristics of eGFR equations (including those using cystatin C) are welcome to better identify CKD and decide on its management, although existing data have shown that the effects of SGLT2 inhibitors on kidney function are consistent, regardless of the clearance method used [20-22].

Acknowledgements The authors would like to thank the participants, their families and all investigators involved in the VERTIS CV study; the list of investigators can be found with the primary publication [11]. Medical writing and/or editorial assistance was provided by M. Hammad and I. Norton, both of Scion (London, UK). This assistance was funded by Merck Sharp \& Dohme Corp., a subsidiary of Merck \& Co., Inc., (Kenilworth, NJ, USA) and Pfizer Inc. (New York, NY, USA).

Data availability The data sharing policy, including restrictions, of Merck Sharp \& Dohme Corp., a subsidiary of Merck \& Co., Inc., (Kenilworth, NJ, USA), is available at http://engagezone.msd.com/ds documentation.php. Requests for access to the clinical study data can be submitted through the EngageZone site or via email to: dataaccess@ merck.com.

Funding Medical writing and/or editorial assistance for this letter was sponsored by Merck Sharp \& Dohme Corp., a subsidiary of Merck \& Co., Inc. (Kenilworth, NJ, USA) and Pfizer Inc. (New York, NY, USA). The VERTIS CV study was funded by Merck Sharp \& Dohme Corp., a subsidiary of Merck \& Co., Inc. (Kenilworth, NJ, USA), in collaboration with Pfizer Inc. The sponsors were involved in the study design, collection, analysis and interpretation of data, as well as data checking of information provided in the manuscript.

Authors' relationships and activities DZIC has received consulting fees or speaking honorarium, or both, from Bristol-Myers Squibb, Novo Nordisk, Mitsubishis-Tanabe, MAZE, Janssen, Bayer, Boehringer Ingelheim, Eli Lilly, AstraZeneca, Merck \& Co., Inc., Prometic and Sanofi, and has received operating funds from Janssen, Boehringer Ingelheim-Eli Lilly, Sanofi, AstraZeneca, Novo Nordisk and Merck \& Co., Inc., Prometic and Sanofi, and has received operating funds from Janssen, Boehringer Ingelheim, Eli Lilly, Sanofi, AstraZeneca and Merck \& Co., Inc. BC has received fees for the following activities: advisory boards for AstraZeneca, Merck Sharp \& Dohme Corp., a subsidiary of Merck \& Co., Inc. (Kenilworth, NJ, USA), Novo Nordisk, Sanofi and Servier; and speaker bureau for AstraZeneca, Eli Lilly, Merck Sharp \& Dohme Corp., a subsidiary of Merck \& Co., Inc. (Kenilworth, NJ, USA), Novo Nordisk, Sanofi and Takeda. FC has received fees from Abbott, AstraZeneca, Bayer, Boehringer Ingelheim, Bristol-Myers Squibb, Merck Sharp \& Dohme, Novo Nordisk and Pfizer, as well as research grants from Swedish Research Council, Swedish Heart \& Lung Foundation and the King Gustav V and Queen Victoria Foundation. SD-J has led clinical trials for AstraZeneca, Novo Nordisk, Inc. and Boehringer Ingelheim, has received fees from AstraZeneca, Boehringer Ingelheim, Janssen, Merck \& Co., Inc. and Sanofi, and holds equity interests in Jana Care, Inc. and Aerami Therapeutics. DKM has had leadership roles in clinical trials for AstraZeneca, Boehringer Ingelheim, CSL Behring, Eisai, Esperion, GlaxoSmithKline, Janssen, Lexicon, Merck \& Co., Inc., Novo Nordisk and Sanofi USA, and has received consultancy fees from AstraZeneca, Bayer, Boehringer Ingelheim, Lilly USA, Merck \& Co., Inc., Pfizer, Novo Nordisk, Metavant, Afimmune and Sanofi. RP has received the following fees (directed to his institution): speaker fees 
from Novo Nordisk; consulting fees from Merck \& Co., Inc., Novo Nordisk, Pfizer, Sanofi, Scohia Pharma Inc. and Sun Pharmaceutical Industries; and grants from Lexicon Pharmaceuticals, Hanmi Pharmaceuticals Co., Novo Nordisk, Poxel SA and Sanofi. WJS has received fees for membership of the ertugliflozin advisory board of Merck \& Co., Inc. (Kenilworth, NJ, USA). RF is an employee and shareholder of Pfizer Inc. MM is an employee of MSD UK, and may own stock and/or stock options in Merck \& Co., Inc. (Kenilworth, NJ, USA). AP is an employee of Merck Sharp \& Dohme Corp., a subsidiary of Merck \& Co., Inc. (Kenilworth, NJ, USA) and may own stock and/or stock options in Merck \& Co., Inc. (Kenilworth, NJ, USA). CPC reports grants and personal fees from Pfizer Inc. and from Merck \& Co., Inc., during the conduct of the study. CPC also reports grants and personal fees from Amgen, Boehringer Ingelheim, Bristol-Myers Squibb and Janssen, grants from Better Therapeutics, Daiichi Sankyo Novo Nordisk, and personal fees from Aegerion/Amryt, Alnylam, Amarin, Applied Therapeutics, Ascendia, Corvidia, HLS Therapeutics, Innovent, Kowa, Sanofi, Eli Lilly, Lexicon, Rhoshan, and Sanofi outside the submitted work. CPC serves on Data and Safety Monitoring Boards for the Veterans Administration, Applied Therapeutics and Novo Nordisk.

Contribution statement All authors were responsible for drafting the article and/or revising it critically for important intellectual content. All authors approved the version to be published.

Open Access This article is licensed under a Creative Commons Attribution 4.0 International License, which permits use, sharing, adaptation, distribution and reproduction in any medium or format, as long as you give appropriate credit to the original author(s) and the source, provide a link to the Creative Commons licence, and indicate if changes were made. The images or other third party material in this article are included in the article's Creative Commons licence, unless indicated otherwise in a credit line to the material. If material is not included in the article's Creative Commons licence and your intended use is not permitted by statutory regulation or exceeds the permitted use, you will need to obtain permission directly from the copyright holder. To view a copy of this licence, visit http://creativecommons.org/licenses/by/4.0/.

\section{References}

1. Groothof D, Post A, Gans ROB, Bakker SJL (2021) Ertugliflozin, renoprotection and potential confounding by muscle wasting. Diabetologia. https://doi.org/10.1007/s00125-021-05614-0

2. Cherney DZI, Heerspink HJL, Frederich R et al (2020) Effects of ertugliflozin on renal function over 104 weeks of treatment: a post hoc analysis of two randomised controlled trials. Diabetologia 63(6):1128-1140. https://doi.org/10.1007/s00125-020-05133-4

3. Lambers Heerspink HJ, de Zeeuw D, Wie L, Leslie B, List J (2013) Dapagliflozin a glucose-regulating drug with diuretic properties in subjects with type 2 diabetes. Diabetes Obes Metab 15(9):853-862. https://doi.org/10.1111/dom.12127

4. Kosugi R, Nakatani E, Okamoto K, Aoshima S, Arai H, Inoue T (2019) Effects of sodium-glucose cotransporter 2 inhibitor (dapagliflozin) on food intake and plasma fibroblast growth factor 21 levels in type 2 diabetes patients. Endocr J 66(8):677-682. https://doi.org/10.1507/endocrj.EJ19-0013

5. Lundkvist $\mathrm{P}$, Sjöström CD, Amini S, Pereira MJ, Johnsson E, Eriksson JW (2017) Dapagliflozin once-daily and exenatide onceweekly dual therapy: A 24-week randomized, placebo-controlled, phase II study examining effects on body weight and prediabetes in obese adults without diabetes. Diabetes Obes Metab 19(1):49-60. https://doi.org/10.1111/dom.12779

6. Sugiyama S, Jinnouchi H, Kurinami N et al (2018) Dapagliflozin reduces fat mass without affecting muscle mass in type 2 diabetes. $\mathrm{J}$ Atheroscler Thromb 25(6):467-476. https://doi.org/10.5551/jat. 40873

7. Tobita H, Sato S, Miyake T, Ishihara S, Kinoshita Y (2017) Effects of dapagliflozin on body composition and liver tests in patients with nonalcoholic steatohepatitis associated with type 2 diabetes mellitus: a prospective, open-label, uncontrolled study. Curr Ther Res Clin Exp 87:13-19. https://doi.org/10.1016/j.curtheres.2017. 07.002

8. Bolinder J, Ljunggren Ö, Kullberg J et al (2012) Effects of Dapagliflozin on Body Weight, Total Fat Mass, and Regional Adipose Tissue Distribution in Patients with Type 2 Diabetes Mellitus with Inadequate Glycemic Control on Metformin. J Clin Endocrinol Metab 97(3):1020-1031. https://doi.org/10.1210/jc. 2011-2260

9. Fadini GP, Bonora BM, Zatti G et al (2017) Effects of the SGLT2 inhibitor dapagliflozin on HDL cholesterol, particle size, and cholesterol efflux capacity in patients with type 2 diabetes: a randomized placebo-controlled trial. Cardiovasc Diabetol 16(1):42

10. Post A, Groothof D, Eisenga MF, Bakker SJL (2020) Sodiumglucose cotransporter 2 inhibitors and kidney outcomes: true renoprotection, loss of muscle mass or both? J Clin Med 9(5): 1603. https://doi.org/10.3390/jcm9051603

11. Wolf VLW, Breder I, de Carvalho LSF et al (2021) Dapagliflozin increases the lean-to total mass ratio in type 2 diabetes mellitus. Nutr Diabetes 11(1):17

12. List JF, Woo V, Morales E, Tang W, Fiedorek FT (2009) Sodiumglucose cotransport inhibition with dapagliflozin in type 2 diabetes. Diabetes Care 32(4):650-657. https://doi.org/10.2337/dc08-1863

13. Heerspink HJL, Jongs N, Chertow GM et al (2021) Effect of dapagliflozin on the rate of decline in kidney function in patients with chronic kidney disease with and without type 2 diabetes: a prespecified analysis from the DAPA-CKD trial. Lancet Diabetes Endocrinol 9(11):743-754

14. Chertow GM, Vart P, Jongs $\mathrm{N}$ et al (2021) Effects of dapagliflozin in stage 4 chronic kidney disease. J Am Soc Nephrol 32(9):23522361. https://doi.org/10.1681/ASN.2021020167

15. Heerspink HJL, Stefansson BV, Correa-Rotter R et al (2020) Dapagliflozin in patients with chronic kidney disease. N Engl J Med 383(15):1436-1446. https://doi.org/10.1056/ NEJMoa2024816

16. Packer M, Anker SD, Butler J et al (2020) Cardiovascular and renal outcomes with empagliflozin in heart failure. N Engl J Med 383: 1413-1424. https://doi.org/10.1056/NEJMoa2022190

17. Cannon CP, Pratley R, Dagogo-Jack S et al (2020) Cardiovascular outcomes with ertugliflozin in type 2 diabetes. N Engl J Med 383: 1425-1435. https://doi.org/10.1056/NEJMoa2004967

18. Cherney DZI, Charbonnel B, Cosentino F et al (2021) Effects of ertugliflozin on kidney composite outcomes, renal function and albuminuria in patients with type 2 diabetes mellitus: an analysis from the randomised VERTIS CV trial. Diabetologia 64:12561267. https://doi.org/10.1007/s00125-021-05407-5

19. Tattersall J, Dekker F, Heimbürger O et al (2011) When to start dialysis: updated guidance following publication of the Initiating Dialysis Early and Late (IDEAL) study. Nephrol Dial Transplant 26(7):2082-2086. https://doi.org/10.1093/ndt/gfr168

20. Cherney DZI, Dekkers CCJ, Barbour SJ et al (2020) Effects of the SGLT2 inhibitor dapagliflozin on proteinuria in non-diabetic patients with chronic kidney disease (DIAMOND): a randomised, double-blind, crossover trial. Lancet Diabetes Endocrinol 8(7):582593. https://doi.org/10.1016/S2213-8587(20)30162-5

21. van Ruiten CC, van der Aart-van der Beek AB, IJzerman RG et al (2021) Effect of exenatide twice daily and dapagliflozin, alone and 
in combination, on markers of kidney function in obese patients with type 2 diabetes: A prespecified secondary analysis of a randomized controlled clinical trial. Diabetes Obes Metab 23(8): 1851-1858. https://doi.org/10.1111/dom. 14410

22. Cherney DZ, Perkins BA, Soleymanlou N et al (2014) Renal hemodynamic effect of sodium-glucose cotransporter 2 inhibition in patients with type 1 diabetes mellitus. Circulation 129(5):587597. https://doi.org/10.1161/CIRCULATIONAHA.113.005081

Publisher's note Springer Nature remains neutral with regard to jurisdictional claims in published maps and institutional affiliations. 